\title{
Haplotypes and mutations of the PAH locus in Egyptian families with PKU
}

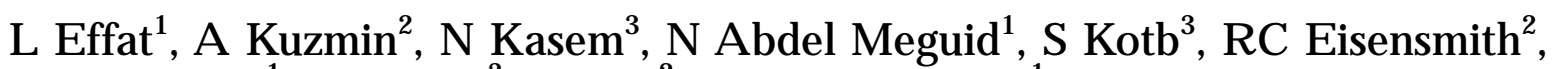 \\ SA Temtamy ${ }^{1}$, S R ushdi ${ }^{3}$, S Woo $^{2}$ and M El-A wady ${ }^{1}$ \\ ${ }^{1} \mathrm{D}$ epartment of H uman G enetics, N ational Research Center, E gypt \\ ${ }^{2} B$ aylor College of M edicine, Houston, Texas, USA \\ ${ }^{3} \mathrm{D}$ epartment of $\mathrm{H}$ uman $\mathrm{G}$ enetics M edical R esearch Institute, A lexandria U niversity, E gypt
}

\begin{abstract}
A high degree of molecular heterogeneneity at the phenylalanine hydroxylase (PA H) locus was established by examining RFLP haplotypes and PAH mutations in the families of $13 \mathrm{E}$ gyptians with phenylketenouria (PKU). Thirteen different haplotypes were unequivocally determined in these kindreds. $H$ aplotypes $1.8,3.9,4.3,7.8,22.11,27.6$, and 52.8 were found segregating with normal chromosomes, whilst haplotypes $1.8,5.9,23.8,32.8$, the newly assigned 73.9, and two as yet incomplete but novel haplotypes were found segregating with the mutant chromosomes. There was no particular preference for a single haplotype among normal or mutant chromosomes. $\mathrm{N}$ ine different mutations were also identified among the 26 alleles. IV S 10nt1lg (8/26), IV S 2nt5g-c (4/26), R 261Q (3/26), R 176X (2/26), Y 206D (2/26), S231P (2/26), Y 198fs [593-614del22bp]; (2/26), G 46fs [136/137delG ]; (1/26), and E 178G (1/26). Six of these mutations (IV S 2nt5g-c, R 176X, Y 198fs, R 261Q, S231P, and IV S 10nt1lg) are common to other Mediterranean populations. Two mutations not previously reported in the Mediterranean basin were also observed (Y 206D and G 46fs). These intriguing preliminary findings confirm IV S 10nt1lg as a major mutation among Mediterranean mutations and demonstrate the need for a more comprehensive study of $A$ rab populations to confirm the uniqueness of the two novel mutations to the Egyptian population.
\end{abstract}

Keywords: Mutation; PAH locus; PKU diagnosis; Haplotypes; polymorphism population genetics

\section{Introduction}

Phenylketonuria (PKU), one of the most common inborn errors of amino acid metabolism, is associated with the presence of a large number of molecular defects in the phenylalanine hydroxylase $(\mathrm{PA} \mathrm{H})$ gene. $^{1}$ $M$ ost of these mutations greatly reduce or abolish the

Correspondence: Laila $\mathrm{E}$ ffat $\mathrm{PhD}$, D epartment of Human Genetics, National Research Center, Tahrir Street, Dokki 12622, Cairo. Fax: 202 3559169; E-mail: reffat@tc.cac.edu.eg R eceived 14 J une 1996; revised 9 September 1998; accepted 17 September 1998 activity of the liver PAH enzyme, ${ }^{2}$ which normally converts the essential dietary amino acid $\mathrm{L}$-phenylalanine to $\mathrm{L}$-tyrosine. ${ }^{3} \mathrm{In}$ individuals affected with PKU, levels of phenylalanine in the blood and other tissues increase significantly due to the PAH deficiency. ${ }^{4}$ If these high levels are not detected very early in infancy and significantly reduced by administration of a special diet low in phenylalanine, severe and irreversible mental retardation ensues. ${ }^{5}$

Isolation of the gene and the determination of the sequence of full length PA H CDNA ${ }^{6}$ not only permitted the characterisation of restriction fragment length 
polymorphism (RFLP) haplotypes and their application in prenatal diagnostic procedures, ${ }^{7}$ but also facilitated the initial mutational analysis at the PA H locus. Subsequent studies in European, Mediterranean and A sian communities disclosed a variety of PAH mutations. $^{8,9}$

Prenatal diagnosis of PKU is an important adjunct to dietary treatment for the prevention of the ensuing mental retardation, as the limited availability of the low-phenylalanine diet and poor compliance are still major problems in E gypt. The identification of different haplotypes and mutations present at the PA $\mathrm{H}$ locus in Egyptians offers the possibility of proper diagnosis of probands, facilitates the identification of carriers and provides genetic counselling regarding the relative risk of the disease in their offspring. In addition prenatal diagnosis of PKU can be performed in families whenever mutational or haplotype analysis is informative.

\section{Materials and Methods}

\section{Subjects}

E ighteen affected individuals from 13 families presenting with mental retardation were diagnosed at the $\mathrm{N}$ ational $\mathrm{R}$ esearch Center to have hyperphenylalaninaemia. Elevated levels of phenylalanine in serum were diagnosed by Guthrie test, and confirmed by thin layer chromatography and H PLC.

\section{Determination of Normal and Mutant Haplotypes (HTs) at the PAH Locus}

L eucocytic D NA was extracted from venous blood withdrawn from affected individuals, parents and normal sibs, using the salting-out technique. Haplotype $(\mathrm{HT})$ analysis was based on the eight polymorphic R FLPNNTR sites scattered throughout the PA H gene. ${ }^{10}$ Five restriction sites ( $\mathrm{Bgll}$ I, Pvulla $\& b$, $\mathrm{Mspl}$, and $\mathrm{Xmnl}$ ) were analysed by $P C R$ amplification followed by restriction endonuclease digestion as described by Dworniczak et al. ${ }^{11-14}$ The VNTR site was amplified according to Golstov et al. ${ }^{15}$ Both the E co RI and E co RV were analysed by Southern blotting as described by Woo. ${ }^{16}$ The haplotype assignments were determined from the polymorphic sites according to the tabulation of $\mathrm{E}$ isensmith and Woo. ${ }^{17}$ Complete HT analysis was not possible in some families, since both parents were not available. In other families, heterozygosity of all family members for one or two of the sites prevented the assignment of definitive haplotypes.

\section{Mutation Detection}

B ased on the haplotype data and the mutation/haplotype associations in Southern Europeans, Turks and Palestinian A rabs, seven mutations common to these populations ( $\mathrm{L} 48 \mathrm{~S}$, E 221G，R 252W，R 261Q，E 280K，P281L and IV S 10 nt 11g) were screened by PCR amplification of the exons 2, 6, 7, and 11. This amplification was followed by restriction enzyme digestion as described by $\mathrm{E}$ iken et al. ${ }^{18}$ Following this initial screening, all exons were examined using a combination of
DGGE and direct sequencing as described by Kuzmin et al. $^{19}$

\section{Results}

\section{Polymorphic Haplotypes}

Eight loci spread throughout the PA H structural gene were analysed for polymorphism to identify the different HTs present in the Egyptian PKU kindreds. A high degree of heterogeneity was found among both normal and mutant alleles. Within the fully informative families HTs1.8 $(n=1), 3.9(n=1), 4.3(n=1), 7.8(n=2)$, $22.11(n=1), 27.6(n=1), 52.8(n=1)$ were observed on normal chromosomes, whilst haplotypes $1.8(n=1)$, $5.9(n=1), 23.8(n=1), 32.8(n=1)$ were found on the mutant chromosome. Mutant chromosomes also contained the novel HT $73.9(n=2)$ and two incomplete, but tentatively novel haplotypes. Half the patients studied appeared to be the result of consanguineous marriages, as the probands were homozygous for both haplotype and mutation. In non-consanguineous marriages different $\mathrm{HT}$ combinations were always found (eg H T 1.8/H T 32.8; H T 23.8/H T 5.9). Complete H T analysis was not possible in some families, since not all parents were available. In other families, heterozygosity of all family members for one or two of the sites prevented the assignment completion of the haplotypes.

\section{Mutations}

The mutational screening analysis revealed nine different mutations IV S $10 \mathrm{nt11g}(8 / 26)$, IV S 2 nt5g-c (4/26), R 261Q (3/26), R 176X (2/26), Y 206D (2/26), S231P (2/26), Y 198fs [593-614del22bp]; (2/26), G 46fs [136/137delG ]; (1/26), and E 178G (1/26) with a high frequency of the IVS $10 \mathrm{nt11g}$ in particular (Table 1). Six of these mutations, namely IV S 2 nt5g-c, R 176X,

Table 1 Point mutations from the studied sample

\begin{tabular}{llr}
\hline M utation & Frequency & \\
\hline 1 IV S 10 nt1 1g & $8 / 26$ & $30.8 \%$ \\
2 IV S2 nt5g-c & $4 / 26$ & $15.4 \%$ \\
3 R 261Q & $3 / 26$ & $11.5 \%$ \\
4 R 176X & $2 / 26$ & $7.7 \%$ \\
5 Y 206D & $2 / 26$ & $7.7 \%$ \\
6 S231P & $2 / 26$ & $7.7 \%$ \\
7 Y 198fs & $2 / 26$ & $7.7 \%$ \\
8 G 46fs & $1 / 26$ & $3.8 \%$ \\
9 E 178G & $1 / 26$ & $3.8 \%$ \\
10 not identified & $1 / 26$ & $3.8 \%$ \\
Total & $26 / 26$ & $100 \%$ \\
\hline
\end{tabular}


Table 2 VNTR, haplotype, genotype relationship

\begin{tabular}{|c|c|c|c|}
\hline Family & Haplotype & VNTR & G enotype \\
\hline $\bar{A}$ & HT $1 * / \mathrm{HT}^{2} 32^{*}$ & $8 * / 8 *$ & R 176X /R 261Q \\
\hline $\mathrm{B}$ & HTN \#*/HTN\# & $9 * / 9 *$ & IV S2nt5/IV S2nt5 \\
\hline $\mathrm{C}$ & HT $23^{*} / \mathrm{HT}^{*}$ & $8 * / 9 *$ & G 46fs/l V S2nt \\
\hline D & $\mathrm{HT} ? / \mathrm{HT} ?$ & $3^{*} / 3^{*}$ & Y 206D / 206D \\
\hline $\mathrm{E}$ & HTN \#/ / T N \# & $9 * / 9 *$ & E 178G / Y 198fs \\
\hline $\mathrm{F}$ & HT?/HT? & $7 * / 7 *$ & IV S10nt11g/IV S10nt11g \\
\hline G & HT?/HT? & $9 * / 9 *$ & IV S2nt5/? \\
\hline $\mathrm{H}$ & HT?/HT? & $7 * / 7 *$ & IV S10nt11g/IV S10nt11g \\
\hline 1 & HT?/HT? & $9 * / 8^{*}$ & Y 198fs/R 176X \\
\hline & HTN\#/HTN\# & $7 * / 7 *$ & IV S10nt11g/IV S10nt11g \\
\hline K & HT?/HT? & $7 * / 7 *$ & IV S10nt11g/IV S10nt11g \\
\hline$M$ & HT?/HT? & $3 * / 3 *$ & R 261Q /R 261Q / \\
\hline $\mathrm{N}$ & HT?/HT? & $11 * / 11 *$ & $\mathrm{~S} 213 \mathrm{P} / \mathrm{S} 213 \mathrm{P}$ \\
\hline
\end{tabular}

Y 198fs, R 261Q, S231P, and IVS 10nt11g are common M editerranean mutations. The IV S 2nt5g-c was associated with two different $\mathrm{HT}$ backgrounds, HT 5.9 and the new HT 73.9 (Table2). Two new mutations that have not been reported elsewhere in the Mediterranean basin, G 46fs (136/137delG ) and Y 206D were found among our sample, on one and two alleles, respectively. Further screening in the area will determine the haplotype associations of these novel mutations and resolve whether they are unique to E gypt or common to other A rab populations.

\section{Discussion}

The rich variety of polymorphism and mutations at the PAH locus makes it a useful tool in molecular population genetics. And many investigators have delineated PA H haplotypes and established their distribution among normal and mutant chromosomes in different populations (see Konecki ${ }^{20}$ for a review). A lthough most of these studies have shown the PA H locus to be very heterogenous, with more than 46 distinct HTs in E urope alone, ${ }^{21}$ only six HTs $(1,2,3,4,5$ and 7) were identified on $76 \%$ of all normal chromosomes, ${ }^{15}$ with predominance of HTs 1 and 4 . These latter two haplotypes have been detected in all populations studied thus far, with only a few exceptions. ${ }^{20}$ In the Mediterranean population most relevant to the present study (I taly, Turkey, Palestenian A rabs), H Ts 1, 4 and to a lesser extent 7 were also predominant among normal chromosomes. ${ }^{22}$ Based on our preliminary findings, the distribution of normal haplotypes in the Egyptian population is not significantly different from that of other M editerranean populations, with H Ts 1.8, $3.9,4.3$ and 7.8 present on more than half of the normal chromosomes of known haplotypes. The other normal
HTs (22.11, 27.6 and 52.8) are rare in both Egyptian and $\mathrm{E}$ uropean populations.

$\mathrm{H}$ aplotype and mutational studies of mutant PAH chromosomes have also demonstrated a high degree of molecular heterogeneity in Egyptian PKU families. $\mathrm{H}$ aplotype studies identified seven different mutant haplotypes and nine different mutations among the 26 mutant chromosomes analysed. In comparison with $\mathrm{Dr}$ $\mathrm{H}$ ashem's study ${ }^{23}$ suggesting the rarity of IV S 10nt1lg in Egypt, this study showed a high frequency of this mutation (8/26) in Table 1. The relative predominance of this mutation among Southern European mutations has also been demonstrated in several other Mediterranean populations, including Spain, I taly, G reece, Turkey and Palestinian, Moroccan and other Jewish populations of the region. ${ }^{22}$ The heterogeneity at the PA H locus in Egyptian PKU families may easily be explained on the basis of Egypt's unique geographic location and rich historical past. Egypt lies at the crossroads of three continents and since ancient Pharaonic times, has been the interest of many conquerors. The inclusion of E gypt as a part of the $O$ smani E mpire for over 400 years produced many social intermarriages between the two populations, intertwining the genetic backgrounds. Thus, the high frequency of the IVS 10nt1lg mutation may reflect these close familial relationships that exist between E gyptians and Turks.

Five of the other mutations detected in this study, IVS 2nt5g-c, R 176X, Y 198fs, S231P and R 261Q, have been observed in certain other $M$ editerranean populations, although at low frequencies. Of these mutations, IVS 2 nt $5 \mathrm{~g}$-c was the most frequent. At a relative frequency of $4 / 26$, it was the second most prevalent mutation identified in this study and much more frequent in E gypt than in any other population in which it has been detected. ${ }^{24}$ This mutation was present on two different HT backgrounds, HT 5.9 and HT 73.9; IVS 2 nt5g-c has previously been reported to be associated with $\mathrm{HTs} 1$ and $5 .{ }^{24} \mathrm{~A} s$ in other populations, HT 1 in Egypt contained multiple mutations; R $261 \mathrm{Q}$ and R176X were both present on this haplotype background (Table 2). The association between $\mathrm{R} 261 \mathrm{Q}$ and HT 1 was not exclusive, as this mutation was also observed on a single chromosome of HT 32.8. The haplotype association of R 176X in the Sicilian population was not reported. Of the remaining mutations previously identified in other populations, E 178G and Y 198fs were each present on the incomplete, but tentatively novel haplotype also containing the 9-copy VNTR. The haplotype association of S231P could not 
be established. In addition to these previously identified mutations, two novel mutations were detected among these E gyptian PKU families. These were $G 46 f 5$ and $Y 206 D$, present on one and two chromosomes, respectively. $\mathrm{G} 46 \mathrm{fs}$ was associated with $\mathrm{HT}$ 23.7. Further screening in the area will resolve whether these mutations are unique or common in the A rab population.

These studies defining the molecular bases of PKU in the E gyptian population are critically important for the management of this disease in this developing country. The information gleaned from these studies can facilitate the choice of priorities for mutational screening, thus saving time, effort and expense. Identification of the mutations responsible for PKU can assist in providing accurate information to the families with regard to prognosis in the absence of treatment and response to treatment if available. These data can also be used to perform carrier detection and offer reproductive counselling to the apparently normal sibs. Finally, prenatal diagnosis can be offered at an early stage, thus avoiding the birth of an affected child facing the prospects of profound mental retardation in a society where low-phenylalanine dietary products are scarce or absent.

\section{References}

1 Eisensmith RC, Woo SLC: Molecular basis of phenylketonuria and related hyperphenylalaninemias: mutations and polymorphisms in the human phenylalanine hydroxylase gene. Hum M ut 1992; 1: 13-23.

2 Jervis GA : D eficiency of phenylalanine oxidizing system. Pro Soc Exp Biol Med 1953; 82: 514-515.

3 Trefz FK, Schmidt $H$, B artholome $K$, M ahle $M$, M atthis $P$, Pecht $G$ : D ifferential diagnosis and significance of various hyperphenylalaninemias. In: B ickel $\mathrm{H}$, Wachtel $\mathrm{U}$ (eds). Inherited Diseases of A mino A cid M etabolism. Thieme: Stuttgart, 1985, pp 86-100.

4 Scriver CR, Kaufman S, E isensmith RC, Woo SL C: The hyperphenylalaninemias. I $n$ : Scriver CR, B eaudet A L, Sly WS, Valle D (eds). The M etabolic and M olecular B ases of Inherited D isease, 7th edn. M cG raw-H ill: N ew Y ork, 1995; vol. 1, pp 1015-1075.

5 Smith I: Phenylketonuria. In: H osking G, M urphy G (eds). Prevention of $M$ ental $H$ andicap. R oyal Society of Medicine International Congress and Symposium Series 112. R oyal Society of M edicine: L ondon, 1987, pp 59-61.

$6 \mathrm{~K}$ wok S, Ledley FD, Dilella A G, R obson KJH, Woo SL C: Nucleotide sequence of a full-length CDNA clone of human phenylalanine hydroxylase. B iochemistry 1985; 24: 556-561.
7 Lidsky AS, Ledley FD, Dilella AG et al: Extensive restriction site polymorphism at the human phenylalanine hydroxilase locus and application in prenatal diagnosis of phenylketonuria. A m J Hum G enet 1985; 37: 619-634.

8 Scriver CR, John SWM, R ozen R, Eisensmith R C, Woo SLC: A ssociations between population, PKU mutations and RFLP haplotypes at the phenylalanine hydroxilase locus: an overview. Brain D ysfunct 1992; 6: 11-25.

9 E isensmith RC, Woo SL C: M olecular genetics of phenylketonuria: from molecular anthropology to gene therapy. In: Hall JC, Dunlap JC, Friedman T, G ianelli F (eds). A dvances in G enetics. A cademic Press: San Diego, 1995, vol. 32, pp 199-272.

10 Chakraborty R, Lidsky A S, D aiger SP et al: Polymorphic DNA haplotypes at the human phenylalanine hydroxilase locus and their relationship with phenylketonuria. H um G enet 1987; 76: 40-46.

11 D worniczak B, Wedemeyer N, Horst J : PCR detection of the Pvull (E a) R FLP at the human phenylalanine hydroxilase (PA H) locus. Nucleic A cids R es 1991; 19: 1958.

12 D worniczak $B$, Wedemeyer $N$, H orst J : PCR detection of the B gll I R FL P at the human phenylalanine locus. Nucleic A cids Res 1991; 19: 1958

13 Wedemeyer N, D worniczak B, H orst J : PCR detection of the M spl ( $\mathrm{A}$ a) R FLP at the human phenylalanine hydroxilase (PA H) locus. Nucleic A cids R es 1991; 19: 1959.

14 Goltsov A A, E isensmith R C, Woo SL C: D etection of the $\mathrm{Xmnl} R F L P$ at the human phenylalanine hydroxilase (PA H) locus by PCR. Nucleic A cids Res 1992; 20: 927.

15 Goltsov AA, Eisensmith RC, Konecki DS, LichterKonecki U, Woo SLC: A ssociation between mutations and a VNTR in the human phenylalanine hydroxilase gene. A m J H um G enet 1992; 51: 627-636.

16 Woo SLC: Prenatal diagnosis and carrier detection of classic phenylketonuria by gene analysis. Pediatrics 1984; 74: 412-422.

17 E isensmith R C, Woo SL C: U pdated listing of haplotypes at the human phenylalanine hydroxilase locus. A $\mathrm{m} \mathrm{J} \mathrm{H} \mathrm{um}$ G enet 1992; 51: 1445-1448.

18 Eiken H G, O dland E, B oman $H$, Skjelkvale L, Engebresten $L F, A$ pold $]$ : A pplication of natural and amplification created restriction sites for the diagnosis of PKU mutations. N ucleic A cids Res 1991; 7: 1427-1430.

19 Kuzmin A, E isensmith RC, Sergeeva NA, Goltsov A, Swartz E, Woo SLC: Complete spectrum of PA H mutations in tataria. E ur J H um G enet 1995; 3: 246-255.

20 Konecki DS, Lichter-Konecki U: The phenylketonuria locus: current knowledge about alleles and mutations of the phenylalanine hydroxilase gene in various populations. H um G enet 1991; 87: 377-388.

21 Daiger SP, Chakraborty R, Reed L et al: Polymorphic DNA haplotypes at the human phenylalanine hydroxilase locus in E uropean families with phenylketonuria (PKU ). A m J H um G enet 1989; 45: 310-3318.

22 Kleiman S, Smadar A, Vanagite $L$ et al: Origins of hyperphenylalaninemia in I srael. E ur J H um G enet 1994; 2: $24-34$.

23 D esviat L R, Perez B, U garte M : Phenylketonuria in Spain: R FL P haplotypes and linked mutations. H um G enet 1993; 92: 254-258.

24 Nowachi $\mathrm{P}$ et al: The PA H M utation A nalysis Consortium D atabase: update 1996. Nucleic A cids Res 1997; 25(1) 139-142. 